\title{
MicroRNA-222 regulates the viability of fibroblasts in hypertrophic scars via matrix metalloproteinase 1
}

\author{
YI ZHANG ${ }^{1,2}$, XIAOHUA LIN ${ }^{2}$, LI ZHANG ${ }^{2}$, WEILONG HONG ${ }^{3}$ and KANG ZENG ${ }^{1}$ \\ ${ }^{1}$ Department of Dermatology, Nanfang Hospital, Southern Medical University, Guangzhou, \\ Guangdong 510515; Departments of ${ }^{2}$ Dermatology and ${ }^{3}$ Surgery Lab, The First Affiliated Hospital, \\ Wenzhou Medical University, Wenzhou, Zhejiang 325000, P.R. China
}

Received September 17, 2016; Accepted April 10, 2017

DOI: $10.3892 / \mathrm{etm} .2017 .5634$

\begin{abstract}
The present study aimed to determine the expression of microRNA (miR)-222 in hypertrophic scar (HS) tissues, and investigate the regulatory mechanism of miR-222 in HS. A total of 36 patients diagnosed with HS between August 2013 and May 2016 were included in the present study. HS tissues and HS-adjacent tissues were collected from patients. Primary fibroblasts were obtained from HS tissue. Reverse transcription-quantitative polymerase chain reaction was used to measure mRNA levels of matrix metalloproteinase 1 (MMP1) and miR-222. Western blotting was conducted to determine MMP1 expression and an MTT assay was performed to measure the viability of fibroblasts. A dual luciferase reporter assay was used to identify the binding of miR-222 to MMP1 mRNA. It was demonstrated that MMP1 serves a role in HS at the transcription level and that increased MMP1 expression inhibited the viability of fibroblasts. miR-222 serves a regulatory role in HS by targeting its target gene MMP1 and regulates the expression of MMP1 by binding to its 3'-untranslated region. The decreased expression of miR-222 suppresses the viability of fibroblasts by regulating MMP1 expression. The present study demonstrated that the downregulation of MMP1 in HS tissues is associated with the upregulation of miR-222 expression. miR-222 may therefore regulate the viability of fibroblasts in HS and the expression of related proteins via MMP1.
\end{abstract}

\section{Introduction}

Following wound healing in human skin, a hypertrophic scar (HS) often forms at the wound site. This is essentially a type of fibrotic disease (1). The formation of a pathological scar primarily occurs due to an imbalance between collagen

Correspondence to: Dr Kang Zeng, Department of Dermatology, Nanfang Hospital, Southern Medical University, 1838 Guangzhou Road, Guangzhou, Guangdong 510515, P.R. China

E-mail: ycv888@126.com

Key words: hypertrophic scar, microRNA-222, matrix metalloproteinase 1 deposition and degradation (2). The current clinical treatments for HS mainly include non-surgical methods (local compression, scar silicone film sticking, laser therapy, cryotherapy, corticosteroids local injection and radiotherapy) and surgical excision of scar tissue (3-5). However, these methods have limitations, and cannot achieve satisfactory therapeutic effects, and so new treatments continue to emerge (6). Proline hydroxylase is the rate-limiting enzyme in the synthesis of collagen. An environment containing high levels of lactic acid induced by hypoxia following trauma may enhance the activity of proline hydroxylase and promote collagen deposition $(7,8)$. Matrix metalloproteinases (MMPs) that can degrade extracellular matrix and collagen serve important roles in the reactions of cells to their external environment. MMPs hydrolyze proteins and interrupt the synthesis of extracellular matrix, as well as regulate interactions between cells and the external environment. Therefore, MMPs affect the biochemistry, migration, proliferation and survival of cells, and inhibiting MMP activity promotes scar formation (9-11). MMP1, also known as fibroblast type I collagenase, is a key enzyme that degrades collagen in human skin (12) and serves important roles in HS $(13,14)$.

MicroRNA (miR) molecules are small non-coding RNA molecules 22 nucleotides long and certain miRs inhibit MMP1 expression and affect the fibrosis of skin cells (15). It has been demonstrated that miR-222 targets MMP1 and regulates the biological functions of squamous cancer cells of the tongue (16). However, to the best of our knowledge, the regulation of miR-222 on fibroblasts in HS has not been previously reported. In the present study, the expression of MMP1 mRNA and protein in HS tissues was assessed and the association between MMP1 and miR-222 expression was evaluated.

\section{Patients and methods}

Patients. A total of 36 patients diagnosed with HS at the First Affiliated Hospital of Wenzhou Medical University (Wenzhou, China) between August 2013 and May 2016 were included in the present study. Both HS tissues (experimental group) and HS-adjacent tissues (control group) were collected from all patients. The tissues were resected from the patients and frozen at $-80^{\circ} \mathrm{C}$ in liquid nitrogen for further use. The 36 patients included 20 males and 16 females, aged between 
17 and 61 years (median age, 45.6 years). None of the patients had used hormones, traditional Chinese medicine, radiotherapy or chemotherapy within one year prior to the current study. In all patients, HS were formed by scalds or burns and met the Patient and Observer Scar Assessment Scale classification criteria $(17,18)$. All procedures were approved by the Ethics Committee of Wenzhou Medical University. Written informed consent was obtained from all patients or their families.

Cells. To obtain primary fibroblasts, HS tissues and HSadjacent tissues were washed with $0.1 \mathrm{M}$ phosphate-buffered saline ( $\mathrm{pH}$ 7.4) three times. The tissues were then digested with $0.25 \%$ dispase overnight to remove the layer of skin. Following homogenization, tissues were digested with $0.1 \%$ type I collagenase (C-0130; Sigma-Aldrich; Merck KGaA, Darmstadt, Germany) at $37^{\circ} \mathrm{C}$ by shaking for $3 \mathrm{~h}$. Digestion was terminated by mixing the samples with an equal volume of low-glucose Dulbecco's modified Eagle's medium (DMEM; SH30023.01B; Hyclone; GE Healthcare Life Sciences, Logan, UT, USA) supplemented with $10 \%$ fetal bovine serum (H30084.03; Hyclone, GE Healthcare Life Sciences). Following centrifugation at $300 \mathrm{x} \mathrm{g}$ and room temperature for $10 \mathrm{~min}$, the supernatant was discarded and fibroblasts were resuspended in high-glucose DMEM. Fibroblasts were seeded onto culture plates with a diameter of $100 \mathrm{~mm}$ at a density of $4 \times 10^{4} / \mathrm{cm}^{2}$ and cultured at $37^{\circ} \mathrm{C}$ under $5 \% \mathrm{CO}_{2}$ and $100 \%$ humidity for $24 \mathrm{~h}$. The medium was initially replaced after $24 \mathrm{~h}$ and subsequently replaced every 2 days. Cells were passaged when reaching confluence.

One day prior to transfection, log-phase fibroblasts $\left(3 \times 10^{5}\right.$ cells/well) were seeded onto 24 -well plates containing F12/DMEM medium supplemented with $10 \%$ fetal bovine serum without antibiotics. To silence MMP1, the MMP1 small-interfering RNA (siRNA) was used to transfect fibroblasts. For transfection of fibroblasts with antagomiR-222, cells were divided into a control group (untreated; only for MTT assay), a negative control (NC) group transfected with negative plasmids and an miR-222 mimic group. When cells reached $70 \%$ confluence, $1 \mu \mathrm{l}$ MMP1 plasmids, siRNA or antagomiR-222 (both from Sangon Biotech, Co., Ltd., Shanghai, China) and $1 \mu 1$ Lipofectamine ${ }^{\circledR} 2000$ (Thermo Fisher Scientific, Inc., Waltham, MA, USA) were added to two individual vials each containing $50 \mu \mathrm{l}$ Opti-Mem medium (Thermo Fisher Scientific, Inc.). After $5 \mathrm{~min}$, the liquids in the two vials were mixed before standing still for $20 \mathrm{~min}$ at room temperature. Then, the mixture was added to the cells for $6 \mathrm{~h}$ incubation at $37^{\circ} \mathrm{C}$. The medium was changed to fresh F12/DMEM medium supplemented with $10 \%$ fetal bovine serum and fibroblasts were cultured at $37^{\circ} \mathrm{C}$ for $48 \mathrm{~h}$ prior to use.

Reverse transcription-quantitative polymerase chain reaction (RT-qPCR). Tissues (100 mg) were ground into powder using liquid nitrogen prior to addition of $1 \mathrm{ml}$ TRIzol (10606ES60; Shanghai Yeasen Biotechnology, Co., Ltd., Shanghai, China) prior to lysis. Following lysis, total RNA was extracted using the phenol chloroform method (19). RNA purity was determined by A260/A280 using ultraviolet spectrophotometry (Nanodrop ND1000; Thermo Fisher Scientific, Inc.). Then, reverse transcription of $1 \mu \mathrm{g}$ RNA was performed using the TIANScript II
cDNA first-strand synthesis kit (KR107; Tiangen Biotech, Co., Ltd., Beijing, China) and cDNA was stored at $-20^{\circ} \mathrm{C}$. For miR, an miRcute miRNA cDNA First-strand Synthesis kit (KR201; Tiangen Biotech, Co., Ltd.) was used to synthesize cDNA. The primers used for qPCR of MMP1 were as follows: MMP1 forward, 5'-GGACACCAACTATTGCTTCAG-3' and reverse, 5'-ATGTCCTTGGGGTATCCGTGTAG-3'; GAPDH forward, 5'-TCCTGTGGCATCCACGAAACT-3' and reverse, 5'-GAA GCATTTGCGGTGGACGAT-3'. The PCR reaction system (25 $\mu \mathrm{l}$ ) was composed of $10 \mu \mathrm{l}$ qRT-PCR-mix [SuperReal PreMix (SYBR-Green); FP204; Tiangen, Biotech, Co., Ltd.], $0.5 \mu \mathrm{l}$ upstream primer, $0.5 \mu \mathrm{l}$ downstream primer, $2 \mu \mathrm{l} \mathrm{cDNA}$ and $7 \mu 1 \mathrm{ddH}_{2} \mathrm{O}$. The PCR conditions were: Initial denaturation at $95^{\circ} \mathrm{C}$ for $3 \mathrm{~min} ; 39$ cycles of denaturation at $94^{\circ} \mathrm{C}$ for $20 \mathrm{sec}$, annealing at $58^{\circ} \mathrm{C}$ for $20 \mathrm{sec}$ and elongation at $72^{\circ} \mathrm{C}$ for $20 \mathrm{sec}$, and final elongation at $72^{\circ} \mathrm{C}$ for 10 min using an iQ5 Real-Time PCR Detection system (Bio-Rad Laboratories, Inc., Hercules, CA, USA). The $2^{-\Delta \Delta C q}$ method (20) was used to calculate the relative expression of MMP1 mRNA against GAPDH.

To determine the expression of miR-222, the miRcute miRNA qPCR detection kit (FP401; Tiangen, Biotech, Co., Ltd.) was selected and U6 was used as an internal reference. The primers used were as follows: miR-222 forward, 5'-CGC AGCTACATCTGGCTACTG-3' and reverse, 5'-GTGCAG GGTCCGAGGT-3'; U6 forward, 5'-CGCTTCGGCAGCACA TATAC-3' and reverse, 5'-CAGGGGCCATGCTAATCTT-3'. The PCR protocol was as follows: Initial denaturation at $95^{\circ} \mathrm{C}$ for $5 \mathrm{~min}$; 40 cycles of denaturation at $95^{\circ} \mathrm{C}$ for $30 \mathrm{sec}$ and annealing at $60^{\circ} \mathrm{C}$ for $35 \mathrm{sec}$, and final elongation at $72^{\circ} \mathrm{C}$ for $20 \mathrm{sec}$ using the iQ5 Real-Time PCR Detection system. The $2^{-\Delta \Delta C q}$ method was used to calculate the relative expression of miR-222 against U6.

Western blotting. Tissues (100 mg) were ground using liquid nitrogen and mixed with $200 \mu$ l precooled Radioimmunoprecipitation assay lysis buffer $(600 \mu 1 ; 50 \mathrm{mM}$ Tris-base, $1 \mathrm{mM}$ EDTA, $150 \mathrm{mM} \mathrm{NaCl}, 0.1 \%$ sodium dodecyl sulfate, $1 \%$ TritonX-100, $1 \%$ sodium deoxycholate; Beyotime Institute of Biotechnology, Shanghai, China) for 30 min lysis on ice. Subsequently, the mixture was centrifuged at $15,000 \mathrm{x} \mathrm{g}$ for $15 \mathrm{~min}$ at $4^{\circ} \mathrm{C}$. The supernatant was used to determine protein concentration using a bicinchoninic acid protein concentration determination kit [RTP7102; Real-Times (Beijing) Biotechnology Co., Ltd., Beijing, China)]. Protein samples $(20 \mu \mathrm{g})$ were then mixed with sodium dodecyl sulfate loading buffer prior to denaturation in a boiling water bath for $5 \mathrm{~min}$. Subsequently, the samples $(30 \mu \mathrm{g})$ were subjected to $10 \%$ SDS-PAGE. Resolved proteins were transferred to polyvinylidene difluoride membranes on ice $(100 \mathrm{~V}$, $2 \mathrm{~h}$ ) and blocked with 5\% skimmed milk at room temperature for $1 \mathrm{~h}$. Membranes were then incubated with rabbit anti-human MMP1 polyclonal primary antibody (1:500; ab38929) and rabbit anti-human $\beta$-actin primary antibody (1:5,000; ab129348; both from Abcam, Cambridge, UK) at $4^{\circ} \mathrm{C}$ overnight. Following three extensive washes with phosphate-buffered saline and Tween-20 for 15 min each time, the membranes were incubated with polyclonal goat anti-rabbit horseradish peroxidase-conjugated secondary antibody $(1: 3,000$; ab6721; Abcam) for $1 \mathrm{~h}$ at room temperature prior to three washes with phosphate-buffered saline and Tween-20 
for 15 min each time. The membrane was developed using an enhanced chemiluminescence detection kit (Sigma-Aldrich; Merck KGaA) for imaging. Image lab v3.0 software (Bio-Rad Laboratories, Inc.) was used to acquire and analyze imaging signals. The relative content of MMP1 protein was expressed as MMP1/ $\beta$-actin.

MTT assay. Following transfection, cells were seeded into 96-well plates at a density of $2 \times 10^{3}$ cells/well. Each condition was tested in triplicate wells. At 24, 48 and 72 h, $20 \mu 1$ MTT (5 g/l; JRDC000003; JRDUN Biotechnology, Shanghai, China) was added to each well. Dimethyl sulfoxide (150 $\mu \mathrm{l} /$ well) was added to dissolve purple crystals. Following incubation for $4 \mathrm{~h}$ at $37^{\circ} \mathrm{C}$, the absorbance of each well was measured at $490 \mathrm{~nm}$ using a microplate reader (Bio-Rad Laboratories, Inc.) and cell viability curves were plotted.

Dual luciferase reporter assay. Bioinformatic predictions are a powerful tool for the study of miR functions. To determine the regulatory mechanism of MMP1 in HS miRanda (http://www.microrna.org/microrna/home.do), TargetScan (http://www.targetscan.org), PiTa (http://genie. weizmann.ac.il/pubs/mir07/mir07_data.html), RNAhybrid (http://bibiserv.techfak.uni-bielefeld.de/rnahybrid/) and PICTA (http://pictar.mdc-berlin.de) were used to predict the miR molecules that may regulate MMP1. The results suggested that miR-222 may be able to regulate MMP1 (Fig. 1).

According to the results from bioinformatics, wild-type (WT) and mutant seed regions of miR-222 in the 3'-untranslated region (UTR) of the MMP1 gene were chemically synthesized in vitro, added to the SpeI and HindIII restriction sites and then cloned into pMIR-REPORT luciferase reporter plasmids (Ambion; Thermo Fisher Scientific, Inc.). Plasmids $(0.8 \mu \mathrm{g})$ with WT or mutant 3'-UTR DNA sequences were co-transfected with antagomiR-222 (100 nM; Sangon Biotech, Shanghai, China) into 293T cells (Cell Bank of the Chinese Academy of Sciences, Shanghai, China). Following $24 \mathrm{~h}$ cultivation, the cells were lysed using a dual luciferase reporter assay kit (Promega Corporation, Madison, WI, USA) according to the manufacturer's instructions, and fluorescence intensity was measured using a GloMax 20/20 luminometer (Promega Corporation). Using Renilla fluorescence activity as an internal reference, the fluorescence values of each group of cells were measured.

Statistical analysis. The results were analyzed using SPSS 18.0 statistical software (SPSS, Inc., Chicago, IL, USA). The data were expressed as the mean \pm standard deviations and data were tested for normality. Multigroup measurement data were analyzed using one-way ANOVA. In the case of homogeneity of variance, Least Significant Difference and Student-Newman-Keuls methods were used; in the case of heterogeneity of variance, Tamhane's T2 or Dunnett's T3 method was used. $\mathrm{P}<0.05$ was considered to indicate a statistically significant difference.

\section{Results}

MMPl serves a role in HS at the transcription level. To measure the levels of MMP1 mRNA and protein, RT-qPCR

\section{3' ugGGUCAUCGGUC-UACAUCGa 5' hsa-miR-222 $::||::||||||||$ 147:5' uUUUACUAUUUAGAAUGUAGCc 3' MMP1}

Figure 1. Bioinformatics prediction of direct interactions between miR-222 and MMP1. miRanda (http://www.microrna.org/microrna/home.do) TargetScan (http://www.targetscan.org), PiTa (http://genie.weizmann. ac.il/pubs/mir07/mir07_data.html), RNAhybrid (http://bibiserv.techfak. uni-bielefeld.de/rnahybrid/) and PICTA (http://pictar.mdc-berlin.de/) were used to predict miR molecules that may regulate MMP1, and it was determined that miR-222 may be able to regulate MMP1. miR, microRNA; MMP1, matrix metalloproteinase 1 ; hsa, human.

and western blotting were performed, respectively. The data indicated that the expression of MMP1 mRNA and protein in HS tissues was significantly lower than in HS-adjacent tissues $(\mathrm{P}<0.01$ and $\mathrm{P}<0.05$, respectively; Fig. $2 \mathrm{~A}$ and $\mathrm{B})$. These results suggest that MMP1 serves a role in HS at the level of transcription.

Increased MMP1 expression inhibits the viability of fibroblasts. To examine the effect of MMP1 on the viability of fibroblasts, cells were transfected with pcDNAMMP1. RT-qPCR and western blotting indicated that the levels of MMP1 mRNA and protein were significantly increased when MMP1 was overexpressed $(\mathrm{P}<0.01$ and $\mathrm{P}<0.05$, respectively; Fig. 3A and B). In addition, an MTT assay was performed, which demonstrated that the viability of fibroblasts overexpressing MMP1 was significantly inhibited at 48 and $72 \mathrm{~h}$ compared with the control $(\mathrm{P}<0.05$ and $\mathrm{P}<0.01$, respectively; Fig. 4). This indicates that increased MMP1 expression inhibits the viability of fibroblasts.

Expression of miR-222 is elevated in HS. To determine the expression of miR-222 in HS tissues, RT-qPCR was performed. The data demonstrated that the miR-222 level in HS tissues was significantly higher than in HS-adjacent tissues $(\mathrm{P}<0.05$; Fig. 5). The results suggest that expression of miR-222 is elevated in HS.

miR-222 regulates the expression of MMPl by binding to its 3'-UTR. To understand whether miR-222 directly targets MMP1, a dual luciferase reporter assay was performed. The data indicated that transfection with antagomiR-222 and pMIR-REPORT in the WT group led to significantly reduced fluorescence intensity compared with the negative control $(\mathrm{P}<0.01)$, however fluorescence intensity in the mutant group did not differ significantly from that of the negative control ( $\mathrm{P}>0.05$; Fig. 6). These results indicate that miR-222 regulates the expression of MMP1 by binding to the 3'-UTR of MMP1.

Decreased expression of miR-222 suppresses the proliferation of fibroblasts by regulating MMP1 expression. To investigate how miR-222 affects the proliferation of fibroblasts, cells were transfected with antagomiR-222 and an MTT assay was conducted. RT-qPCR indicated that the expression of miR-222 in fibroblasts was significantly decreased following transfection with antagomiR-222 ( $\mathrm{P}<0.01$; Fig. 7A). In addition, expression of MMP1 mRNA significantly increased following transfection with antagomiR-222 ( $\mathrm{P}<0.05$; Fig. 7B). 
A

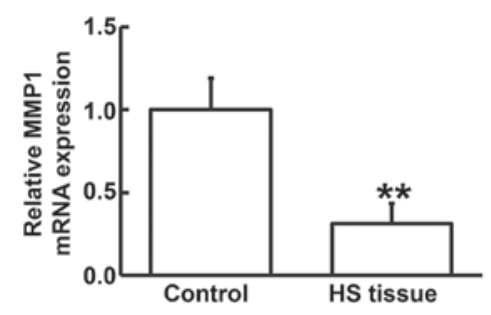

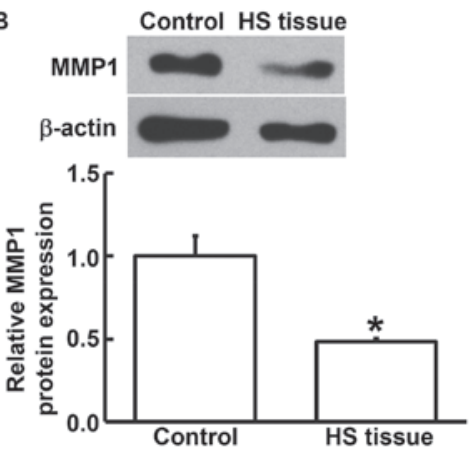

Figure 2. Expression of MMP1 (A) mRNA and (B) protein in HS tissue and HS-adjacent tissue. Reverse transcription-quantitative polymerase chain reaction was used to determine the expression of mRNA and western blotting measured protein expression. Data are presented as the mean \pm standard deviation. ${ }^{*} \mathrm{P}<0.05 ;{ }^{* *} \mathrm{P}<0.01$ vs. control. HS, hypertrophic scar; MMP1, matric metalloproteinase 1.

A

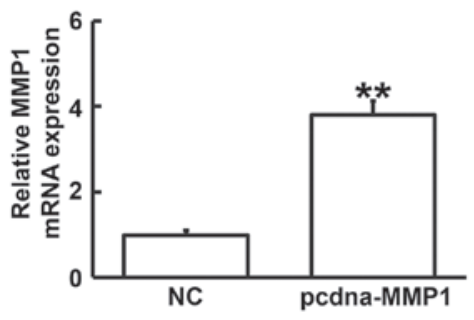

B

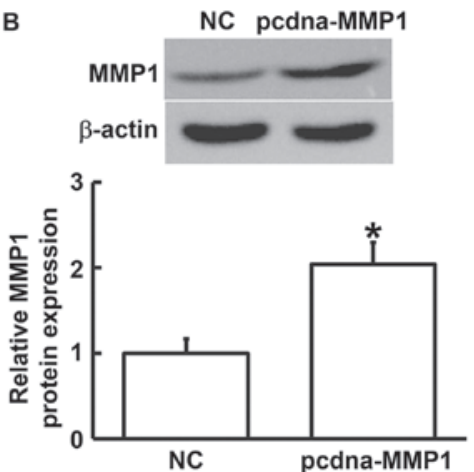

Figure 3. Expression of MMP1 (A) mRNA and (B) protein in fibroblasts prior to and following transfection with pcDNA-MMP1. Reverse transcriptionquantitative polymerase chain reaction was used to determine the expression of mRNA and western blotting was employed to measure protein expression. Data are presented as the mean \pm standard deviation. ${ }^{*} \mathrm{P}<0.05 ;{ }^{* *} \mathrm{P}<0.01$ vs. NC. MMP1, matrix metalloproteinase 1 ; NC, negative control.

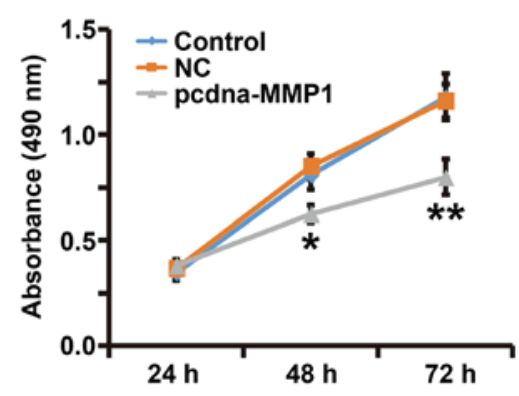

Figure 4. Viability of fibroblasts 24,48 and $72 \mathrm{~h}$ following transfection with pcdna-MMP1. An MTT assay was used to determine the viability of fibroblasts. Absorbance of each well was measured at $490 \mathrm{~nm}$ using a microplate reader and cell viability curves were plotted. Data are presented as the mean \pm standard deviation. ${ }^{*} \mathrm{P}<0.05 ;{ }^{* *} \mathrm{P}<0.01$ vs. control and $\mathrm{NC}$. NC, negative control; MMP1, matrix metalloproteinase 1.

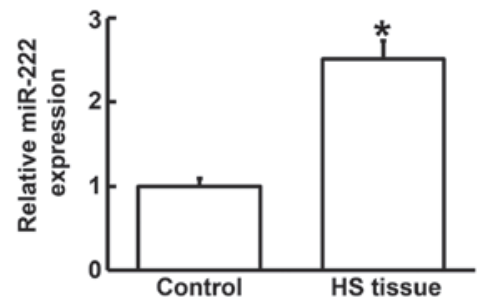

Figure 5. Expression of miR-222 in HS tissue and HS-adjacent tissue. Reverse transcription-quantitative polymerase chain reaction was used to determine the expression of miR-222. Data are presented as the mean \pm standard deviation. " $\mathrm{P}<0.05$ vs. control. miR, microRNA; HS, hypertrophic scar.

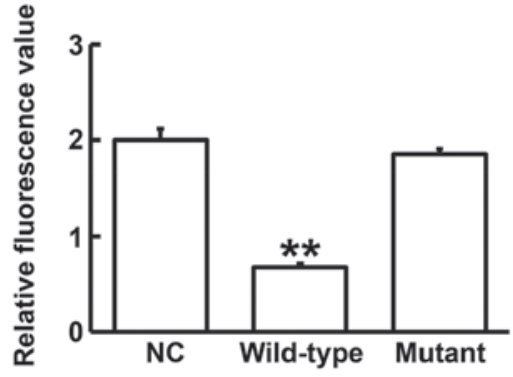

Figure 6. Fluorescence values of 293T cells transfected with wild-type or mutant 3'-untranslated region DNA sequences of MMP1 and antagomiR-222. Dual luciferase reporter assay was used to assess the interaction between miR-222 and MMP1. Data are presented as the mean \pm standard deviation. ${ }^{* *} \mathrm{P}<0.01$ vs. NC. MMP1, matrix metalloproteinase 1 ; miR, microRNA; NC, negative control.

The MTT assay demonstrated that the viability of fibroblasts transfected with antagomiR-222 was significantly reduced at $72 \mathrm{~h}$ compared with the control $(\mathrm{P}<0.01$; Fig. 8). This indicates that increased expression of miR-222 suppresses the proliferation of fibroblasts by regulating the expression of MMP1.

\section{Discussion}

Excessive contracture of scar hyperplasia often leads to various types of abnormal dysfunction (21). Currently, treatments of 

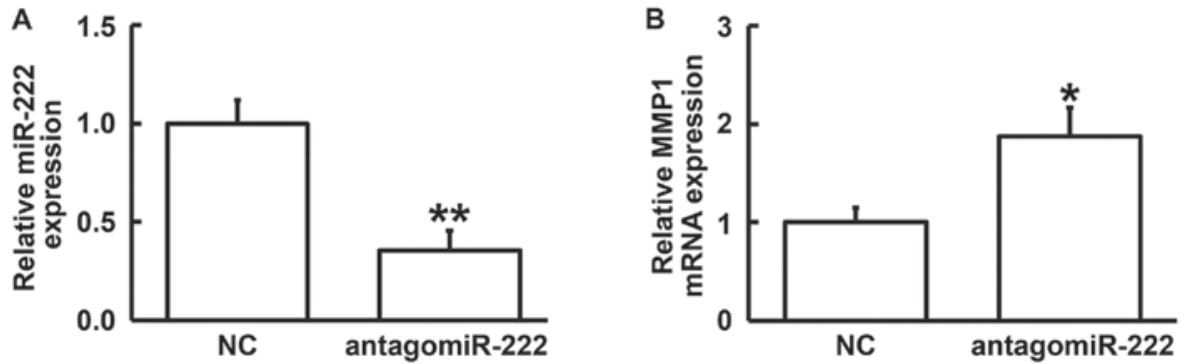

Figure 7. Expression of (A) miR-222 and (B) MMP1 mRNA in fibroblasts prior to and following transfection with antagomiR-222. Reverse transcriptionquantitative polymerase chain reaction was used to determine the expression of miR-222 and MMP-1 mRNA. Data are presented as the mean \pm standard deviation. ${ }^{*} \mathrm{P}<0.05 ;{ }^{* *} \mathrm{P}<0.01$ vs. NC. NC, negative control; MMP1, matrix metalloproteinase $1 ;$ miR, microRNA.

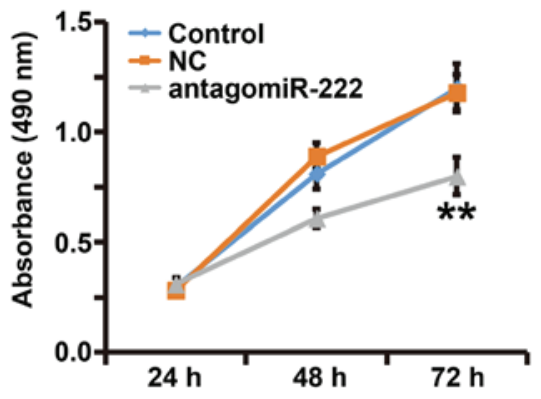

Figure 8 . Viability of fibroblasts 24,48 and $72 \mathrm{~h}$ following transfection with antagomiR-222. The MTT assay was used to determine the viability of fibroblasts. Absorbance of each well was measured at $490 \mathrm{~nm}$ using a microplate reader and cell viability curves were plotted. Data are presented as the mean \pm standard deviation. ${ }^{* *} \mathrm{P}<0.01$ vs. control and $\mathrm{NC}$. NC, negative control; miR, microRNA.

wounds are primarily focused on promoting wound healing and inhibiting excessive scar formation (22-24). However, there are contradictions between these two types of treatments; most notably, growth factors promoting wound healing may also increase the risk of scar formation (25-27). There are currently no effective treatments that promote wound healing and prevent excessive scar formation. The pathological nature of HS is the excessive proliferation of fibroblasts and excessive deposition of extracellular collagen (28). However, the pathogenesis of HS remains unclear. The primary reason for HS formation is a metabolic imbalance between the synthesis and decomposition of extracellular collagen. MMPs are a class of enzymes that are dependent on zinc ions and MMPs secreted by fibroblasts are main enzymes that degrade the extracellular matrix. Inhibition of MMP activity and expression may lead to the onset of diseases characterized by the deposition of extracellular matrix, including connective tissue disease, organ fibrosis disease and atherosclerosis. MMP1 is a key enzyme that degrades collagen in human skin (29). MMP1 activity is regulated by multiple factors, of which the tissue inhibitors of metalloproteinases (TIMPs) are the most important. TIMP1s inhibits the activity of MMP1 by forming a molecular complex (30). Under normal conditions, interactions between MMPs and TIMPs keep the synthesis and degradation of extracellular matrix in a state of dynamic equilibrium. Disruption of this equilibrium may result in insufficient collagen degradation and excessive deposition of extracellular matrix that leads to fibrosis (31). The present study demonstrated that MMP1 expression is downregulated in HS tissues and that the viability of fibroblasts transfected with MMP1 is decreased. These results suggest that the decreased expression of MMP1 may serve an important role in HS.

Previous studies have determined that BTB domain and CNC homolog 1 and long non-coding RNA HOTAIR regulate the expression of MMP1 $(32,33)$. It has been reported that miR-222 regulates the biological function of thyroid follicular epithelial cells via the MEK and tumor necrosis factor-related apoptosis-inducing ligand pathway (34). In addition, miR-222 affects the progression of gastric cancer by regulating its target gene $\mathrm{p} 27^{\mathrm{kip} 1}$ (35). In a mouse model of gastric cancer, abnormal expression of miR-222 has been identified (36). In macrophages associated with ovarian cancer cells, miR-222 regulates the signal transducer and activator of transcription 3 pathway to mediate the polarization of tumor-associated macrophages by targeting suppressor of cytokine signaling 3 (37). In the present study, high expression of miR-222 inhibits the viability of cells. The current study demonstrated that scar formation occurs due to the abnormal proliferation of fibroblasts, in which miR-222 expression is reduced. By contrast, it was demonstrated that upregulation of miR-222 inhibits fibroblast viability. Furthermore, bioinformatics demonstrated that miR-222 targets MMP1. The results of the current study indicate that the reduced level of miR-222 in HS tissues enhances the expression of MMP1 and transfection with antagomiR-222 decreases the viability of fibroblasts. Furthermore, data from the dual luciferase reporter assay indicate that there is direct interaction between miR-222 and MMP1.

There were some limitations in the current study, due to the limited number of cases analyzed and variations in the original locations of the patients who have different diets and lifestyles. In addition, the activity and regulatory mechanism of miR-222 may differ. For example, miR-222 is regulated by other genes (38) and is affected by drug resistance $(39,40)$.

In conclusion, the present study demonstrated that miR-222 negatively regulates MMP1 and suggests that miR-222 may be developed to prevent and treat HS. However further in vitro and in vivo studies are required to determine the actual effect and mechanism of action of miR-222.

\section{Acknowledgements}

The present study was supported by the Health Department of Zhejiang Province-Sponsored Program (no. 2015KYB244). 


\section{References}

1. Niessen FB, Spauwen PH, Schalkwijk J and Kon M: On the nature of hypertrophic scars and keloids: A review. Plast Reconstr Surg 104: 1435-1458, 1999.

2. Tejiram S, Zhang J, Travis TE, Carney BC, Alkhalil A, Moffatt LT, Johnson LS and Shupp JW: Compression therapy affects collagen type balance in hypertrophic scar. J Surg Res 201: 299-305, 2016

3. Sorkin M, Cholok D and Levi B: Scar management of the burned Hand. Hand Clin 33: 305-315, 2017.

4. Cohen BE, Geronemus RG, McDaniel DH and Brauer JA: The role of elastic fibers in scar formation and treatment. Dermatol Surg 43 (Suppl 1): S19-S24, 2017.

5. Wu J, Xu R, Zhan R, Luo G, Niu X, Liu Y, Lee BT, Flury M, Wong $\mathrm{CH}$, Fok $\mathrm{M}$ and Lau JY: Effective symptomatic treatment for severe and intractable pruritus associated with severe burn-induced hypertrophic scars: A prospective, multicenter, controlled trial. Burns 42: 1059-1066, 2016.

6. Wei Y, Li-Tsang CW, Liu J, Xie L and Yue S: 3D-printed transparent facemasks in the treatment of facial hypertrophic scars of young children with burns. Burns 43: e19-e26, 2017.

7. Hunt TK, Conolly WB, Aronson SB and Goldstein P: Anaerobic metabolism and wound healing: An hypothesis for the initiation and cessation of collagen synthesis in wounds. Am J Surg 135: 328-332, 1978.

8. Takahashi Y, Takahashi S, Shiga Y, Yoshimi T and Miura T: Hypoxic induction of prolyl 4-hydroxylase alpha (I) in cultured cells. J Biol Chem 275: 14139-14146, 2000.

9. Baker AH, Edwards DR and Murphy G: Metalloproteinase inhibitors: Biological actions and therapeutic opportunities. J Cell Sci 115: 3719-3727, 2002.

10. Wolfram D, Tzankov A, Pülzl P and Piza-Katzer H: Hypertrophic scars and keloids-a review of their pathophysiology, risk factors and therapeutic management. Dermatol Surg 35: 171-181, 2009.

11. Zhu Z, Ding J, Shankowsky HA and Tredget EE: The molecular mechanism of hypertrophic scar. J Cell Commun Signal 7: 239-252, 2013.

12. Thum T, Chau N, Bhat B, Gupta SK, Linsley PS, Bauersachs J and Engelhardt S: Comparison of different miR-21 inhibitor chemistries in a cardiac disease model. J Clin Invest 121: 461-463, 2011

13. Nabai L, Kilani RT, Aminuddin F, Li Y and Ghahary A: Methotrexate modulates the expression of MMP-1 and type 1 collagen in dermal fibroblast. MolCell Biochem 409: 213-224, 2015.

14. Li Y, Kilani RT, Rahmani-Neishaboor E, Jalili RB and Ghahary A: Kynurenine increases matrix metalloproteinase-1 and -3 expression in cultured dermal fibroblasts and improves scarring in vivo. J Invest Dermatol 134: 643-650, 2014.

15. Kim KH, Jung JY, Son ED, Shin DW, Noh M and Lee TR miR-526b targets 3' UTR of MMP1 mRNA. Exp Mol Med 47: e178, 2015.

16. Liu X, Yu J, Jiang L, Wang A, Shi F, Ye H and Zhou X: MicroRNA-222 regulates cell invasion by targeting matrix metalloproteinase 1 (MMP1) and manganese superoxide dismutase 2 (SOD2) in tongue squamous cell carcinoma cell lines. Cancer Genomics Proteomics 6: 131-139, 2009.

17. van de Kar AL, Corion LU, Smeulders MJ, Draaijers LJ, van der Horst CM and van Zuijlen PP: Reliable and feasible evaluation of linear scars by the patient and observer scar assessment scale. Plast Reconstr Surg 116: 514-522, 2005.

18. Hoogewerf CJ, van Baar ME, Middelkoop E and van Loey NE: Patient reported facial scar assessment: Directions for the professional. Burns 40: 347-353, 2014.

19. Le Bleu HK, Kamal FA, Kelly M, Ketz JP, Zuscik MJ and Elbarbary RA: Extraction of high-quality RNA from human articular cartilage. Anal Biochem 518: 134-138, 2017.

20. Livak KJ and Schmittgen TD: Analysis of relative gene expression data using real-time quantitative PCR and the 2(-Delta Delta C(T)) Method. Methods 25: 402-408, 2001.

21. Gorney M: Scar: The trigger to the claim. Plast Reconstr Surg 117: 1036-1037, 2006

22. Werner $\mathrm{S}$ and Grose R: Regulation of wound healing by growth factors and cytokines. Physiol Rev 83: 835-870, 2003.
23. Berman B, Viera MH, Amini S, Huo R and Jones IS: Prevention and management of hypertrophic scars and keloids after burns in children. J Craniofac Surg 19: 989-1006, 2008

24. Menke MN, Menke NB, Boardman CH and Diegelmann RF: Biologic therapeutics and molecular profiling to optimize wound healing. Gynecol Oncol 111 (2 Suppl): S87-S91, 2008.

25. Younai S, Venters G, Vu S, Nichter L, Nimni ME and Tuan TL: Role of growth factors in scar contraction: An in vitro analysis. Ann Plast Surg 36: 495-501, 1996.

26. Wilgus TA, Ferreira AM, Oberyszyn TM, Bergdall VK and Dipietro LA: Regulation of scar formation by vascular endothelial grow th factor. Lab Invest 88: 579-590, 2008.

27. Grella A, Kole D, Holmes W and Dominko T: FGF2 overrides TGF $\beta 1$-driven integrin ITGA11 Expression in human dermal fibroblasts. J Cell Biochem 117: 1000-1008, 2016.

28. Bai X, He T, Liu J, Wang Y, Fan L, Tao K, Shi J, Tang C, Su L and Hu D: Loureirin B inhibits fibroblast proliferation and extracellular matrix deposition in hypertrophic scar via TGF- $\beta / \mathrm{Smad}$ pathway. Exp Dermatol 24: 355-360, 2015.

29. Scharffetter-Kochanek K, Brenneisen P, Wenk J, Herrmann G, Ma W, Kuhr L, Meewes C and Wlaschek M: Photoaging of the skin from phenotype to mechanisms. Exp Gerontol 35: 307-316, 2000.

30. Pietruszewska W, Bojanowska-Poźniak K and Kobos J: Matrix metalloproteinases MMP1, MMP2, MMP9 and their tissue inhibitors TIMP1, TIMP2, TIMP3 in head and neck cancer: An immunohistochemical study. Otolaryngol Pol 70: 32-43, 2016.

31. Agrinier N, Thilly N, Boivin JM, Dousset B, Alla F and Zannad F: Prognostic value of serum PIIINP, MMP1 and TIMP1 levels in hypertensive patients: A community-based prospective cohort study. Fundam Clin Pharmacol 27: 572-580, 2013.

32. Zhang C, Wang P, Jiang P, Lv Y, Dong C, Dai X, Tan L and Wang Z: Upregulation of lncRNA HOTAIR contributes to IL-1 $\beta$-induced MMP overexpression and chondrocytes apoptosis in temporomandibular joint osteoarthritis. Gene 586: 248-253, 2016.

33. Davudian S, Mansoori B, Shajari N, Mohammadi A and Baradaran B: BACH1, the master regulator gene: A novel candidate target for cancer therapy. Gene 588: 30-37, 2016.

34. Aherne ST, Smyth P, Freeley M, Smith L, Spillane C, O'Leary J and Sheils O: Altered expression of mir-222 and mir-25 influences diverse gene expression changes in transformed normal and anaplastic thyroid cells and impacts on MEK and TRAIL protein expression. Int J Mol Med 38: 433-445, 2016.

35. Lloyd KA, Moore AR, Parsons BN, O'Hara A, Boyce M, Dockray GJ, Varro A and Pritchard DM: Gastrin-induced miR-222 promotes gastric tumor development by suppressing p27kip1. Oncotarget 7: 45462-45478, 2016.

36. Choi B, Yu J, Han TS, Kim YK, Hur K, Kang BC, Kim WH, Kim DY, Lee HJ, Kim VN and Yang HK: Gastric carcinogenesis in the miR-222/221 transgenic mouse model. Cancer Res Treat 49: 150-160, 2017.

37. Ying $X$, Wu Q, Wu X, Zhu Q, Wang X, Jiang L, Chen X and Wang X: Epithelial ovarian cancer-secreted exosomal miR-222-3p induces polarization of tumor-associated macrophages. Oncotarget 7: 43076-43087, 2016

38. Zhao X, Wang P, Liu J, Zheng J, Liu Y, Chen J and Xue Y: Gas5 exerts tumor-suppressive functions in human glioma cells by targeting miR-222. Mol Ther 23: 1899-1911, 2015.

39. Shen H, Wang D, Li L, Yang S, Chen X, Zhou S, Zhong S, Zhao J and Tang J: MiR-222 promotes drug-resistance of breast cancer cells to adriamycin via modulation of PTEN/Akt/FOXO1 pathway. Gene 596: 110-118, 2017.

40. Wang DD, Li J, Sha HH, Chen X, Yang SJ, Shen HY, Zhong SL, Zhao JH and Tang JH: miR-222 confers the resistance of breast cancer cells to Adriamycin through suppression of p27(kip1) expression. Gene 590: 44-50, 2016.

This work is licensed under a Creative Commons Attribution-NonCommercial-NoDerivatives 4.0 International (CC BY-NC-ND 4.0) License. 